\title{
Light Quark Spectroscopy at BESIII
}

\author{
Fang Liu*i \\ Institute of High Energy Physics, Chinese Academy of Sciences, Beijing, China \\ E-mail: liufang@mail.ihep.ac.cn
}

The BESIII experiment at the Beijing Electron and Positron Collider is successfully operating since 2008 and has collected large data samples in the tau-mass region, including the world's largest data samples of $J / \psi, \psi(3686), \psi(3770)$ and $\psi(4040)$ decays. In particular $J / \psi$ and $\psi(3686)$ decays provides a rich and clean environment to study light quark spectroscopy. Many latest experimental searches on the $p \bar{p}$ mass threshold enhancement and $X(1835)$ have been performed by BESIII Collaboration with a high statistical events and provides valuable information that helps to clarify the nature of the states around $1.8 \mathrm{GeV}$. A Partial Wave Analysis (PWA) of the system $\pi^{0} \pi^{0}$ is shown with a focus on the parametrization of the the $\pi \pi$ scattering amplitude. A PWA of the decay $J / \psi \rightarrow \gamma \phi \phi$ shows that the intermediate states in the $\phi \phi$ invariant mass are dominantly $0^{-+}$states, in which the $\eta(2225)$ is confirmed. In this presentation recent results of the light quark spectroscopy will be highlighted.

Flavor Physics and CP Violation,

6-9 June 2016

Caltech, Pasadena CA, USA

${ }^{*}$ Speaker.

$\dagger$ on behalf of BESIII Collaboration 


\section{Introduction}

Glueballs and other resonances with large gluonic components are predicted as bound states by QCD. The lightest (scalar) glueballs is estimated to have a mass in the range from 1 to $2 \mathrm{GeV} / \mathrm{c}^{2}$; pseudoscalar and tensor glueballs are expected at higher masses. Radiative decays of the charmonium provide a glueon rich environment and are therefore regarded as one of the most promising hunting grounds for glueballs and hybrids.

BESIII (Beijing Spectrometer) is a general purpose $4 \pi$ detector at the upgraded BEPCII (Beijing Electron and Positron Collider) that operated in the $\tau$ - charm threshold energy region [1]. Since 2009, it has collected the world's largest samples of $J / \psi, \psi(3686), \psi(3770)$ and $\psi(4040)$ decays. More recently, data were taken in the energy region above $4 \mathrm{GeV}$, where energies up to about $4.6 \mathrm{GeV}$ are accessible. These data are being used to make a variety of interesting and unique studies of light hadron spectroscopy, charmonium spectroscopy, high statistic measurements of charmonium decays and D meson decays.

\section{Light Quark Spectroscopy}

\section{1 $p \bar{p}$ Mass threshold enhancement and $X(1835)$}

An anomalously strong $p \bar{p}$ mass threshold enhancement was first observed by the BESII experiment in the radiative decay process $J / \psi \rightarrow \gamma p \bar{p}[2]$ and was recently confirmed by the BESIII and CLEO-c [3] experiments. Curiously, no apparent corresponding structures were seen in nearthreshold $p \bar{p}$ cross section measurements, in $B$-meson decays [4], in radiative $\psi^{\prime}$ or $\Upsilon \rightarrow \gamma p \bar{p}$ decays [5]. These non-observations disfavor the mass-threshold enhancement attribution to the effects of $p \bar{p}$ final state interactions (FSI) [6, 7, 8].

A number of theoretical speculations have been proposed to interpret the nature of this structure $[6,7,8,9,10,11,12]$. Among them, one intriguing suggestion is that it is due to $p \bar{p}$ bound state, sometimes called baryonium [12], an object with a long history and the subject of many experimental searches [13]. The observation of the $p \bar{p}$ mass threshold enhancement also stimulated many experimental analyses on BESIII experiment.

The Partial Wave Analysis (PWA) of $J / \psi \rightarrow \gamma p \bar{p}$ and $\psi^{\prime} \rightarrow \gamma p \bar{p}$ decays are performed using 225 Million $J / \psi$ and 106 Million $\psi^{\prime}$ events [14]. In $J / \psi$ radiative decays, the events with $M_{p \bar{p}}<2.2 \mathrm{GeV} / c^{2}$ are composed of significant $p \bar{p}$ mass threshold enhancement $X(p \bar{p}), f_{2}(1920)$ and $f_{0}(2100)$ and non-resonant $0^{++}$phase space, as shown in PWA results of Fig. 1. The nearthreshold enhancement $X(p \bar{p})$ is determined to be a $0^{-+}$state. With the inclusion of Julich-FSI effects, the mass, width and product branching fraction (BR) for the $X(p \bar{p})$ are measured to be: $M=$ $1832_{-5}^{+19}$ (stat) ${ }_{-17}^{+18}$ (syst) \pm 19 (model) $\mathrm{MeV} / c^{2}, \Gamma=13 \pm 39$ (stat) $)_{-13}^{+10}$ (syst) \pm 4 (model) $\mathrm{MeV} / c^{2}$ (a total width of $\Gamma<76 \mathrm{MeV} / c^{2}$ at the $90 \%$ C.L) and $\mathscr{B}(J / \psi \rightarrow \gamma X) \times \mathscr{B}(X \rightarrow p \bar{p})=\left(9.0_{-1.1}^{+0.4}\right.$ (stat) ${ }_{-5.0}^{+1.5}($ syst $) \pm 2.3$ (model $\left.)\right) \times 10^{-5}$, respectively. The product BR for $X(p \bar{p})$ in $\psi^{\prime}$ decay is first measured to be $\mathscr{B}\left(\psi^{\prime} \rightarrow \gamma X\right) \times \mathscr{B}(X \rightarrow p \bar{p})=\left(4.57 \pm 0.36\right.$ (stat) ${ }_{-4.07}^{+1.23}$ (syst) \pm 1.28 (model) $) \times 10^{-6}$ and the ratio of product branching fractions for the $X(p \bar{p})$ between $J / \psi$ and $\psi^{\prime}$ radiative decays is $R=\left(5.08_{-0.45}^{+0.71} \text { (stat) }\right)_{-3.58}^{+0.67}$ (syst) \pm 0.12 (model) $) \%$.

Study of the hadronic decays $J / \psi \rightarrow \omega p \bar{p}$ and $\phi p \bar{p}$ may also shed further light on the nature of $X(p \bar{p})$. The decay $J / \psi \rightarrow \omega p \bar{p}$ has been studied [15], using $225.3 \times 10^{6} \mathrm{~J} / \psi$ events accumulated 
at BESIII in 2009. No significant enhancement $X(p \bar{p})$ near the $p \bar{p}$ invariant-mass threshold is observed as shown in Fig. 2. The upper limit of the branching fraction $\mathscr{B}(J / \psi \rightarrow \omega X(p \bar{p}) \rightarrow \omega p \bar{p})$ is determined to be $3.9 \times 10^{-6}$ at the $95 \%$ confidence level, which is suppressed with one order of magnitude comparing to the branching fraction of $J / \psi \rightarrow \gamma X(p \bar{p}) \rightarrow \gamma p \bar{p}$. Another hadronic decay $J / \psi \rightarrow p \bar{p} \phi$ is studied [16] via two decay modes, $\phi \rightarrow K_{S} K_{L}$ and $\phi \rightarrow K^{+} K^{-}$, using a data sample of $1.31 \times 10^{9} \mathrm{~J} / \psi$ events accumulated with the BESIII detector in 2009 and 2012. No evident enhancement $X(p \bar{p})$ near the $p \bar{p}$ mass threshold, is observed as shown in Fig. 3, and the upper limit on the branching fraction of $J / \psi \rightarrow X(p \bar{p}) \phi \rightarrow p \bar{p} \phi$ is determined to be $\mathscr{B}(J / \psi \rightarrow$ $X(p \bar{p}) \phi \rightarrow p \bar{p} \phi)<2.1 \times 10^{-7}$ at the $90 \%$ confidence level, which is much suppressed with two order of magnitude comparing to that of $J / \psi \rightarrow \gamma X(p \bar{p}) \rightarrow \gamma p \bar{p}$.
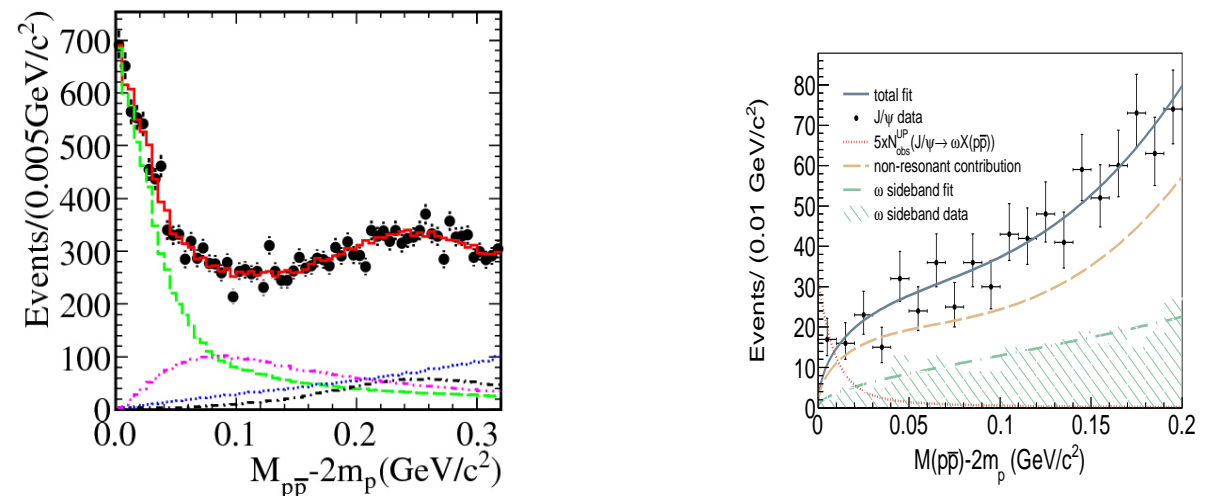

Figure 1: Comparisons of the $p \bar{p}$ invariant mass be- Figure 2: Near-threshold $p \bar{p}$ invariant-mass spectween data and PWA fit projection. The black dots trum. The signal $J / \psi \rightarrow \omega X(p \bar{p}) \rightarrow \omega p \bar{p}$ is dewith error bars are data, the solid histograms show scribed by an acceptance-weighted Breit-Wigner the PWA total projection, and the dashed, dotted, function, and and signal yield is consistent with dash-dotted and dash-dot-dotted lines show the con- zero. The dotted line is the shape of the signal which tributions of the $X(p \bar{p}), 0^{++}$phase space, $f_{0}(2100)$ is normalized to five times the estimated upper limit. and $f_{2}(1910)$, respectively. The hatched area is from the sideband region.
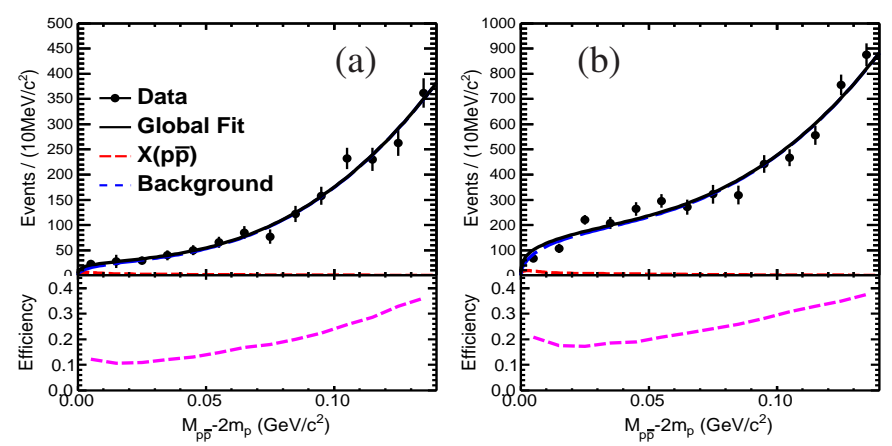

Figure 3: Distributions of $M_{p \bar{p}}-2 m_{p}$ and the fit results corresponding to the upper limit on the branching fraction at the $90 \%$ C.L., the dashed line at the bottom is the efficiency as a function of the $p \bar{p}$ mass, (a) for $\phi \rightarrow K_{S}^{0} K_{L}^{0}$, (b) for $\phi \rightarrow K^{+} K^{-}$.

The observation of the $p \bar{p}$ mass threshold enhancement also stimulated an experimental analy- 
sis of $J / \psi \rightarrow \gamma \pi^{+} \pi^{-} \eta^{\prime}$ decays, in which a $\pi^{+} \pi^{-} \eta^{\prime}$ resonance, the $X(1835)$, was first observed by the BESII experiment [17]. A high statistics data sample collected with BESIII in 2009 provides an opportunity to confirm the existence of the $X(1835)$ and look for possible related states that decay to $\pi^{+} \pi^{-} \eta^{\prime}$. The decay $J / \psi \rightarrow \gamma \pi^{+} \pi^{-} \eta^{\prime}$ is studied [18] combining two $\eta^{\prime}$ decay modes: $\eta^{\prime} \rightarrow \pi^{+} \pi^{-} \eta$ and $\eta^{\prime} \rightarrow \gamma \rho^{0}$. The $X(1835)$ in the $\eta^{\prime} \pi^{+} \pi^{-}$invariant mass as shown in Fig. 4 is confirmed with a statistical significance that is larger than $20 \sigma$. In addition, in the $\pi^{+} \pi^{-} \eta^{\prime}$ invariant mass spectrum, the $X(2120)$ and the $X(2370)$, are observed with statistical significances larger than $7.2 \sigma$ and $6.4 \sigma$, respectively. For the $X(1835)$, the angular distribution of the radiative photon is consistent with expectations for a pseudoscalar, but other spin-parity assignment is not excluded. Then systematic studies of $X(1835)$ are ongoing at BESIII to understand its nature, measurement of its $J^{P C}$ and search for new decays modes is very crucial.
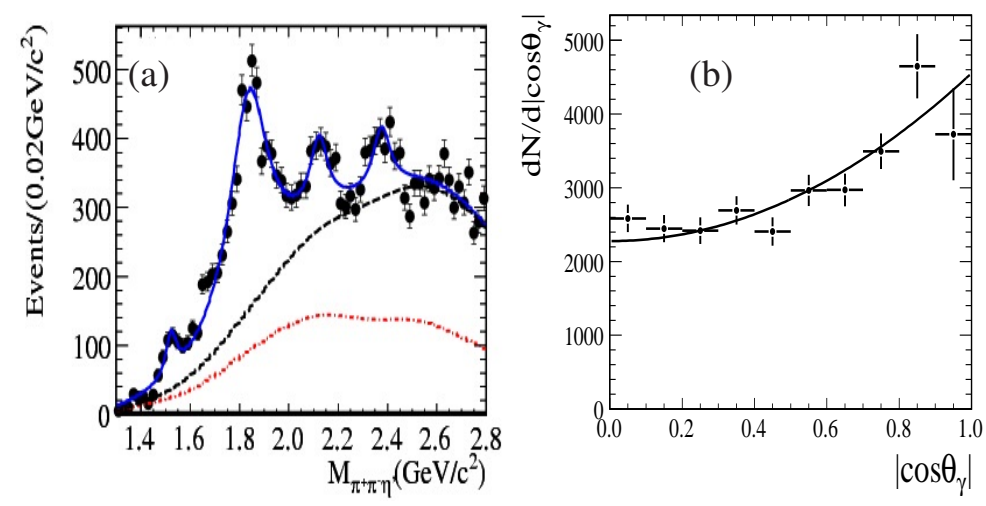

Figure 4: The $\pi^{+} \pi^{-} \eta^{\prime}$ mass spectrum fitting with four resonances (a) and acceptance-corrected $\left|\cos \theta_{\gamma}\right|$ distribution of the $X(1835)$ (b). For (a), the dash-dot line is contributions of non- $\eta^{\prime}$ events and the $\pi^{0} \pi^{+} \pi^{-} \eta^{\prime}$ background for two $\eta^{\prime}$ decay modes and the dash line is contributions of the total background and nonresonant $\pi^{+} \pi^{-} \eta^{\prime}$ process.

We study a new decay mode $J / \psi \rightarrow \gamma K_{S}^{0} K_{S}^{0} \eta$ [19] using data sample of $1.31 \times 10^{9} \mathrm{~J} / \psi$ events collected with the BESIII detector. Figure 5 (a) shows the scatter plot of the invariant mass of $K_{S}^{0} K_{S}^{0}$ versus $K_{S}^{0} K_{S}^{0} \eta$, indicating the structure around $1.85 \mathrm{GeV} / c^{2}$ is strong correlated with $f_{0}(980)$. A partial wave analysis of $J / \psi \rightarrow \gamma K_{S}^{0} K_{S}^{0} \eta$ has been performed in the mass range $M_{K_{S}^{0} K_{S}^{0} \eta}<2.8 \mathrm{GeV} / c^{2}$ after requiring $M_{K_{S}^{0} K_{S}^{0}}<1.1 \mathrm{GeV} / c^{2}$. Figures 5 (b) and (c) are the invariant mass distribution of $K_{S}^{0} K_{S}^{0} \eta$ and $K_{S}^{0} K_{S}^{0}$. Overlaid on the data are the PWA fit projections, as well as the individual contributions from each component. The PWA fit requires a contribution from $X(1835) \rightarrow \gamma K_{S}^{0} K_{S}^{0} \eta$ with a statistical significance larger than $12.9 \sigma$, whether $X(1835) \rightarrow \gamma K_{S}^{0} K_{S}^{0} \eta$ is dominated by $f_{0}(980)$ production. The spin parity of the $X(1835)$ is determined to be $0^{-+}$. The mass and width of the $X(1835)$ are measured to be $1844 \pm 9$ (stat) ${ }_{-25}^{+16}$ (syst) $\mathrm{MeV}$ and $192_{-17}^{+20}(\mathrm{stat})_{-43}^{+62}$ (syst) $\mathrm{MeV}$, respectively. The corresponding product branching fraction is measured to be $\left(3.31_{-0.30}^{+0.33}(\mathrm{stat})_{-1.29}^{+1.96}(\mathrm{syst})\right) \times 10^{-5}$. The mass and width of the $X(1835)$ are consistent with the values obtained from the decay $J / \psi \rightarrow \gamma \pi^{+} \pi^{-} \eta^{\prime}[18]$. These results are all first-times measurements and provide important information to further understand the nature of the $X(1835)$. Another $0^{-+}$state, the $X(1560)$, is also observed in data with a statistical significance larger than $8.9 \sigma$. The mass and width of the $X(1560)$ are consistent with those of the $\eta(1405)$ and $\eta(1475)$ as given in Ref. [20] within $2.0 \sigma$ and $1.4 \sigma$, respectively. 

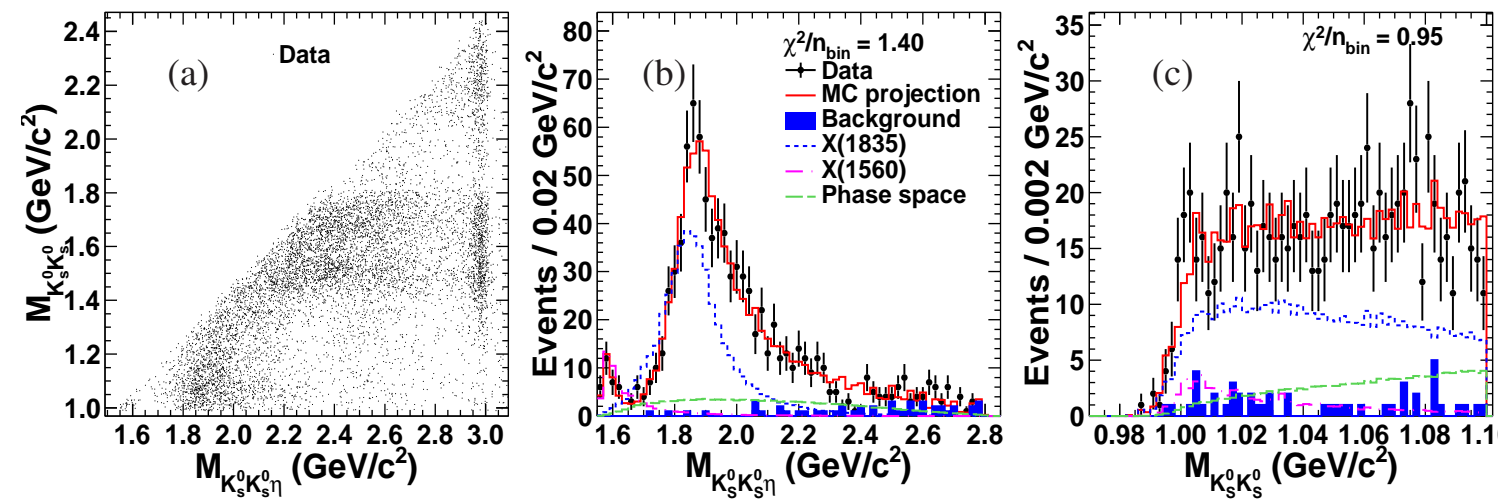

Figure 5: The scatter plot of $M_{K_{S}^{0} K_{S}^{0}}$ versus $M_{K_{S}^{0} K_{S}^{0} \eta}$; Comparisons between data and PWA fit projections. (b), and (c) are the invariant mass distributions of $M_{K_{S}^{0} K_{S}^{0} \eta}$ and $M_{K_{S}^{0} K_{S}^{0}}$. The dots with error bars are data, the solid histograms are the PWA total projections, the shaded histograms are the non- $\eta$ backgrounds estimated by the $\eta$ sideband, and the short-dashed, dash-dotted, and long-dashed histograms show the contributions of $X(1835), X(1560)$, and the non-resonant component, respectively.

The above experimental results stimulate many various theoretical interpretations on the nature of the $X(1835)$ and $X(p \bar{p})[9,10,11,21,22]$, a particularly intriguing one suggests that the two structures originate from a $p \bar{p}$ bound state $[23,24,25,26,27]$. If the $X(1835)$ is really a $p \bar{p}$ bound state, it should have a strong coupling to $0^{-} p \bar{p}$ systems, in which case the line shape of $X(1835)$ at the $p \bar{p}$ mass threshold would be affected by the opening of the $X(1835) \rightarrow p \bar{p}$ decay mode. A study of the $\eta^{\prime} \pi^{+} \pi^{-}$line shape of $X(1835)$ with high statistical precision therefore provides valuable information that helps clarify the nature of the $X(1835)$ and $X(p \bar{p})$. We use a total sample of $1.09 \times 10^{9} \mathrm{~J} / \psi$ decay events [28] accumulated by the BESIII experiment in 2012, and report the observation of a significant abrupt change in slope of the $X(1835) \rightarrow \eta^{\prime} \pi^{+} \pi^{-}$line shape at the $p \bar{p}$ mass threshold in a larger sample of $J / \psi \rightarrow \gamma \eta^{\prime} \pi^{+} \pi^{-}$events [29] collected in the BESIII detector at the BEPCII $e^{+} e^{-}$storage ring. The $\eta^{\prime}$ is also reconstructed in its two major decay modes: $\eta^{\prime} \rightarrow \gamma \pi^{+} \pi^{-}$and $\eta^{\prime} \rightarrow \eta \pi^{+} \pi^{-}(\eta \rightarrow \gamma \gamma)$. Figure 6 shows the $\eta^{\prime} \pi^{+} \pi^{-}$invariant mass spectra after all selection criteria, where peaks corresponding to the $X(1835), X(2120), X(2370)$, $\eta_{c}$, and a structure near $2.6 \mathrm{GeV}$ that has not been seen before are evident for both $\eta^{\prime}$ decays. Thanks to the high statistical precision, an abrupt change in slope of the $X(1835)$ line shape at the $p \bar{p}$ mass threshold is evident in both event samples.

We perform simultaneous fits to the $\eta^{\prime} \pi^{+} \pi^{-}$invariant mass distributions between $1.3 \mathrm{GeV}$ and $2.25 \mathrm{GeV}$ for both selected event samples, and the significant distortion of the $\eta^{\prime} \pi^{+} \pi^{-}$line shape near the $p \bar{p}$ mass threshold cannot be accommodated by an ordinary Breit-Wigner resonance function as shown in Fig. 7 (a). Two typical models for such a line shape are used to fit the data. The first model assumes the state around $1.85 \mathrm{GeV}$ couples with $p \bar{p}$ and the distortion reflects the opening of the $p \bar{p}$ decay channel. The fit result for this model, as shown in Fig. 7(b), yields a strong coupling between the broad structure and the $p \bar{p}$ of $\frac{g_{p \bar{p}}^{2}}{g_{0}^{2}}=2.31 \pm 0.37_{-0.60}^{+0.83}$, with a statistical significance larger than $7 \sigma$ for being non-zero. The pole nearest to the $p \bar{p}$ mass threshold of this state is located at $M_{\text {pole }}=1909.5 \pm 15.9(\text { stat })_{-27.5}^{+9.4}($ syst $) \mathrm{MeV}$ and $\Gamma_{\text {pole }}=273.5 \pm 21.4(\text { stat })_{-64.0}^{+6.1}(\mathrm{syst})$ $\mathrm{MeV}$. The second model assumes the distortion reflects interference between the $X(1835)$ and an- 

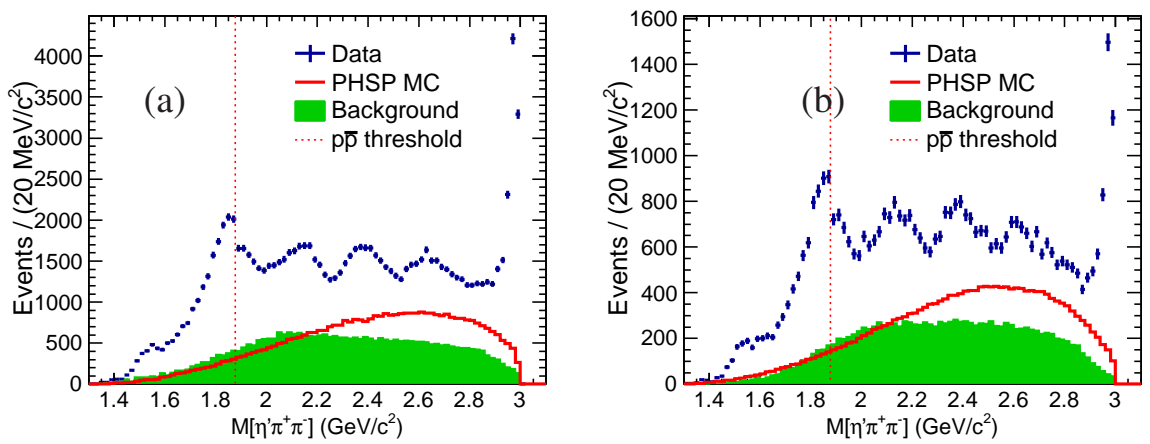

Figure 6: The $\eta^{\prime} \pi^{+} \pi^{-}$invariant mass spectra with the $\eta^{\prime} \rightarrow \gamma \pi^{+} \pi^{-}$channel (a) and $\eta^{\prime} \rightarrow \eta(\rightarrow \gamma \gamma) \pi^{+} \pi^{-}$ channel (b). In both plots, the dots with error bars are data, the shaded histograms are the background, the solid histograms are phase space (PHSP) MC events of $J / \psi \rightarrow \gamma \eta^{\prime} \pi^{+} \pi^{-}$(arbitrary normalization), the dotted vertical line shows the position of $p \bar{p}$ mass threshold.

other resonance with mass close to the $p \bar{p}$ mass threshold. A fit with this model uses a coherent sum of two interfering Breit-Wigner amplitudes to describe the $\eta^{\prime} \pi^{+} \pi^{-}$mass spectrum around $1.85 \mathrm{GeV}$. This fit, as shown in Fig. 7 (c), yields a narrow resonance below the $p \bar{p}$ mass threshold with $M=1870.2 \pm 2.2$ (stat) ${ }_{-0.7}^{+2.3}$ (syst) $\mathrm{MeV}$ and $\Gamma=13.0 \pm 6.1$ (stat $)_{-3.8}^{+2.1}$ (syst) $\mathrm{MeV}$, with a statistical significance larger than $7 \sigma$. With current data, both models fit the data well with fit qualities, and both suggest the existence of a state, either a broad state with strong couplings to $p \bar{p}$, or a narrow state just below the $p \bar{p}$ mass threshold. For the broad state above the $p \bar{p}$ mass threshold, its strong couplings to $p \bar{p}$ suggests the existence of a $p \bar{p}$ molecule-like state. For the narrow state just below $p \bar{p}$ mass threshold, its very narrow width suggests that it be an unconventional meson, most likely a $p \bar{p}$ bound state. So both fits support the existence of a $p \bar{p}$ molecule-like or bound state. With current statistics, more sophisticated models such as a mixture of above two models cannot be ruled out. In order to elucidate further the nature of the states around $1.85 \mathrm{GeV}$, more data are needed to further study $J / \psi \rightarrow \gamma \eta^{\prime} \pi^{+} \pi^{-}$process. Also, line shapes for other decay modes should be studied near the $p \bar{p}$ mass threshold, including further studies of $J / \psi \rightarrow \gamma p \bar{p}$ and $J / \psi \rightarrow \gamma K_{S}^{0} K_{S}^{0} \eta$.

\subsection{Amplitude analysis of the $\pi^{0} \pi^{0}$ system produced in radiative $J / \psi$ decay}

A mass independent amplitude analysis of the $\pi^{0} \pi^{0}$ system in radiative decays is performed [30]. This analysis uses the world's largest sample of its type, collected with the BESIII detector, to extract a piecewise function that describes the scalar and tensor $\pi \pi$ amplitudes in this decay. While the analysis strategy employed to obtain results has complications, namely ambiguous solutions, a large number of parameters, and potential bias in subsequent analysis from non-Gaussian effects, its minimizes systematic bias arising from assumptions about $\pi \pi$ dynamics, and consequently, permits the developments of dynamical models or parameterizations for the data.

The intensities and phase differences for the amplitudes in the fit are presented as a function of $M_{\pi^{0} \pi^{0}}$ in Ref. [30]. Additionally, in order to facilitate the development of models, the intensities and phases of each bin of $M_{\pi^{0} \pi^{0}}$ are given in supplemental materials. These results may be combined with those of similar reactions for a more comprehensive study of the light scalar meson spectrum. 

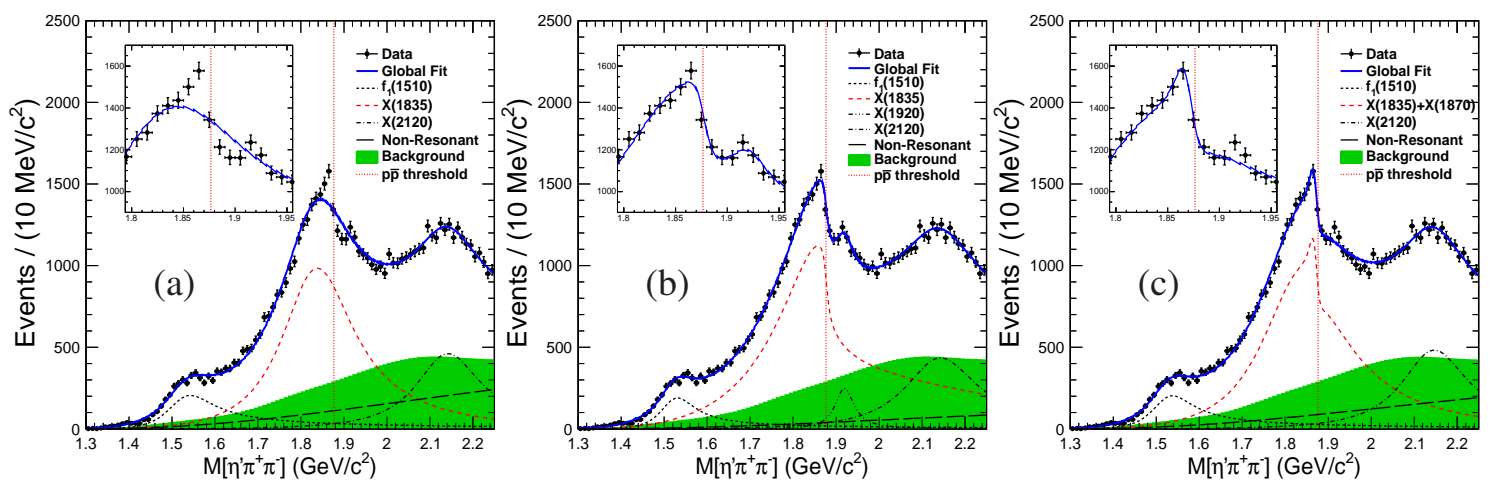

Figure 7: Fit results with simple Breit-Wigner formulae (a), using Flatté formula (b) and using a coherent sum of two Breit-Wigner amplitudes (c), respectively. The dashed dotted vertical line shows the position of $p \bar{p}$ mass threshold, the dots with error bars are data, the solid curves are total fit results, the short-dashed curves the $f_{1}(1510)$, the dash-dot curves the $X(2120)$, and the long-dashed curves are the non-resonant $\eta^{\prime} \pi^{+} \pi^{-}$fit results; the shaded histograms are background events. The dashed curves are the $X(1835)$, the state around $1.85 \mathrm{GeV}$ and the sum of $X(1835)$ and $X(1870)$ for (a) (b) and (c) respectively. The inset shows the data and the global fit between $1.8 \mathrm{GeV}$ and $1.95 \mathrm{GeV}$.

Finally, the branching fraction of radiative $J / \psi$ decays to $\pi^{0} \pi^{0}$ is measured to be $(1.15 \pm 0.05) \times$ $10^{-3}$, where the error is systematic only and the statistical error is negligible. This is the first measurement of this branching fraction.

\subsection{Partial Wave Analysis of $J / \psi \rightarrow \gamma \phi \phi$}

The low lying pseudoscalar glueball is predicted to be around $2.3-2.6 \mathrm{GeV} / c^{2}$ by Lattice QCD [31]. Aside from the $\eta(2225)$, very little is known in the pseudoscalar sector above $2 \mathrm{GeV} / c^{2}$. A partial wave analysis of the decay of $J / \psi \rightarrow \gamma \phi \phi$, as shown in Fig. 8, is performed [32] in order to study the intermediate states. The most remarkable feature of the PWA results is that $0^{-+}$states are dominant. The existence of the $\eta(2225)$ is confirmed and two additional pseudoscalar states, $\eta(2100)$ with a mass of $230_{-35-26}^{+64+77} \mathrm{MeV} / c^{2}$ and a width $250_{-30-164}^{+36+187} \mathrm{MeV} / c^{2}$ and $X(2500)$ with a mass $2470_{-19-23}^{+15+63} \mathrm{MeV} / c^{2}$ and a width $230_{-35-33}^{+64+53} \mathrm{MeV} / c^{2}$, are obtained. The new experimental results are helpful for mapping out pseudoscalar excitations and search for a $0^{-+}$glueball. The three tensors $f_{2}(2100), f_{2}(2300)$ and $f_{2}(2340)$ observing $\pi^{-} p \rightarrow \phi \phi n$ [33] are also observed in $J / \psi \rightarrow \gamma \phi \phi$. Recently, the production rate of the pure gauge tensor glueball in $J / \psi$ radiative decays has been predicted by Lattice QCD [34], which is compatible with the large production rate of the $f_{2}(2340)$ in $J / \psi \rightarrow \gamma \phi \phi$ and $J / \psi \rightarrow \gamma \eta \eta$ [35].

\section{Summary}

With the world's largest sample of $J / \psi, \psi(3686), \psi(3770)$ and $\mathrm{Y}(4040)$. From $e^{+} e^{-}$production, the BESIII experiment made a significant contribution to the study of the light quark spectroscopy. BESIII will continue to run $6-8$ years. Complementary to other experiment, with various production mechanisms, BESIII will give more interesting results and continue shedding light on the nature of hadrons. 

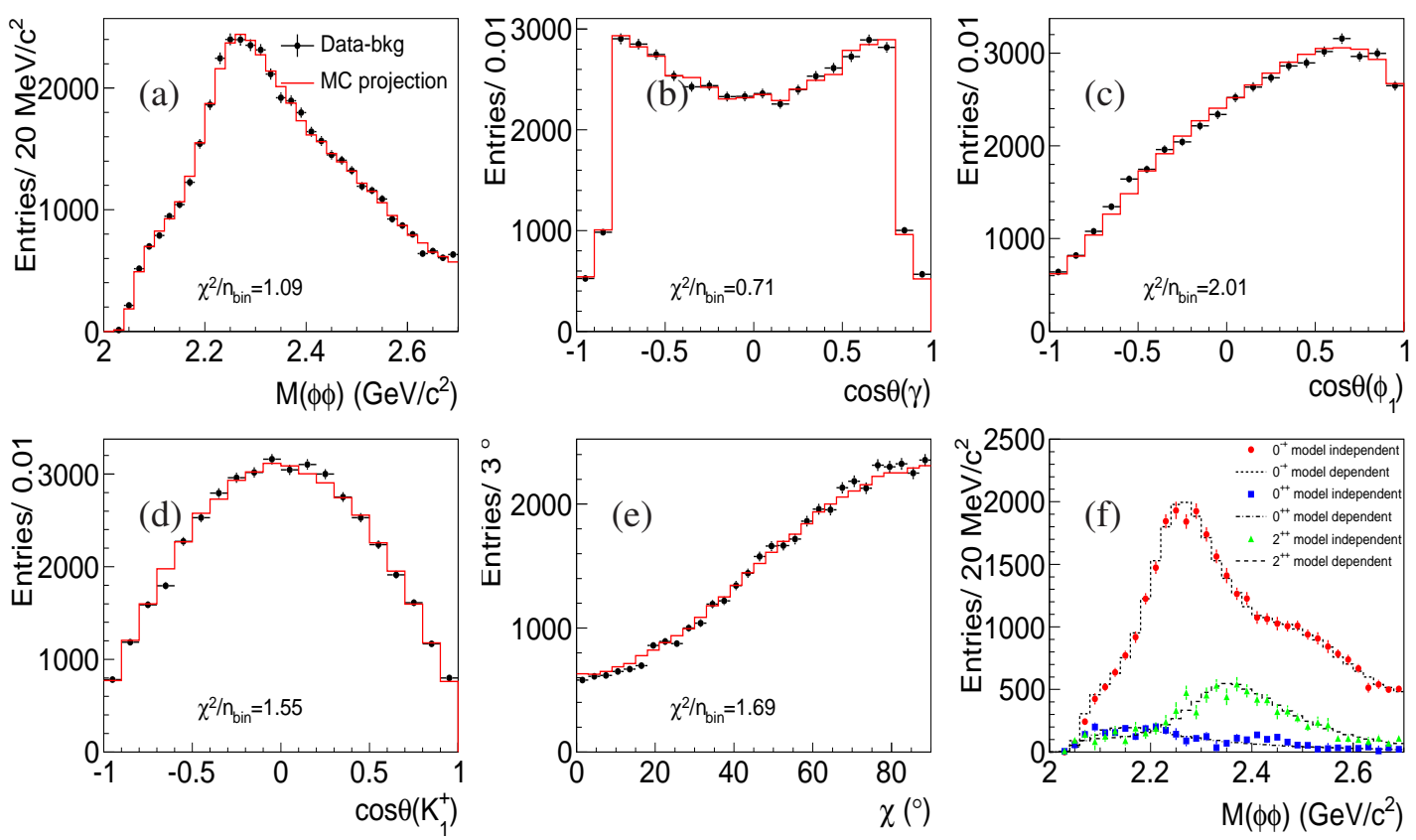

Figure 8: Superposition of data and the PWA fit projections for: (a) invariant mass distributions of $\phi \phi$; (b) $\cos \theta$ of $\gamma$ in the $J / \psi$ rest frame; (c) $\cos \theta$ of $\phi_{1}$ in the $X$ rest frame; (d) $\cos \theta$ of $K^{+}$in the $\phi_{1}$ rest frame; (e) the azimuthal angle between the normals to the two decay planes of $\phi$ in the $X$ rest frame. Black dots with error bars are data with background events subtracted and the solid red lines are projections of the modeldependent fit. (f) Intensities of individual $J^{P C}$ components. The red dots, blue boxes and green triangles with error bars are the intensities of $J^{P C}=0^{-+}, 0^{++}$and $2^{++}$, respectively, from the model-independent fit in each bin. The short-dashed, dash-dotted and long-dashed histograms show the coherent superpositions of the BW resonances with $J^{P C}=0^{-+}, 0^{++}$and $2^{++}$, respectively, from the model-dependent fit.

\section{References}

[1] M. Ablikim et al. (BESIII Collaboration), Nucl. Instrum. Meth. A 614, 345 (2010).

[2] J.Z. Bai et al. (BES Collaboration), Phys. Rev. Lett. 91, 022001 (2003).

[3] M. Ablikim et al. (BESIII Collaboration), Chin.Phys. C34, 421 (2010); J. P. Alexander et al. (CLEO Collaboration), Phys. Rev. D 82, 092002 (2010).

[4] S. Jin, Int. J. Mod. Phys. A 20, 5145 (2005); M.Z. Wang et al., Phys. Rev. Lett. 92, 131801 (2004).

[5] M. Ablikim et al. (BES Collaboration), Phys. Rev. Lett. 99, 011802 (2007); S.B. Athar et al. (CLEO Collaboration), Phys. Rev. D 73, 032001 (2006).

[6] A. Sirbirtsen et al., Phys. Rev. D 71, 054010 (2005).

[7] G. Y. Chen et al., Phys. Lett. B 692, 136 (2010).

[8] B. S. Zou and H. C. Chiang, Phys. Rev. D 69, 034004 (2003).

[9] X. H. Liu et al., Phys. Rev. D 80, 034032 (2009);

[10] N. Kochelev and D. P. Min, Phys. Lett. B 633, 283 (2006).

[11] T. Huang and S. L. Zhu, Phys. Rev. D 73, 014023 (2006). 
[12] A. Datta and P. J. ODonnel, Phys. Lett. B 567, 273 (2003); M. L. Yan et al., Phys. Rev. D 72, 034027 (2005); B. Loiseau et al., Phys. Rev. C 72, 011001 (2005).

[13] E. Klempt et al., Phys. Rep. 368, 119 (2002).

[14] M. Ablikim et al. (BES Collaboration), Phys. Rev. Lett. 108, 112003 (2012).

[15] M. Ablikim et al. (BES Collaboration), Phys. Rev. D 87, 112004 (2013).

[16] M. Ablikim et al. (BES Collaboration), Phys. Rev. D 93, 052010 (2016).

[17] M. Ablikim et al. (BES Collaboration), Phys. Rev. Lett. 95, 262001 (2005).

[18] M. Ablikim et al. (BESIII Collaboration), Phys. Rev. Lett. 106, 072002 (2011).

[19] M. Ablikim et al. (BESIII Collaboration), Phys. Rev. Lett. 115, 091803 (2015).

[20] K. A. Olive et al. (Particle Data Group), Chin. Phys. C 38, 090001(2014).

[21] G. Hao, C. F. Qiao and A. L. Zhang, Phys. Lett. B 642, 53 (2006).

[22] B. A. Li, Phys. Rev. D 74, 034019 (2006).

[23] J. P. Dedonder et al., Phys. Rev. C 80, 045207 (2009).

[24] C. Liu, Eur. Phys. J. C 53, 413 (2008).

[25] Z. G. Wang and S. L. Wan, J. Phys. 34, 505 (2007).

[26] S. L. Zhu and C. S. Gao, Commun. Theor. Phys. 46, 291 (2006).

[27] G. J. Ding, R. G. Ping, and M. L. Yan, Eur. Phys. J. A 28, 351 (2006).

[28] M. Ablikim et al. (BESIII Collaboration), Chin. Phys. C 36, 915 (2012).

[29] M. Ablikim et al. (BESIII Collaboration), Phys. Rev. Lett. 117, 042002 (2016).

[30] M. Ablikim et al. (BESIII Collaboration), Phys. Rev. D 92, 052003 (2015).

[31] C. Amsler and N. A. Tornqvist, Phys. Rev. 389, 61 (2004); E. Klempt and A. Zaitsev, Phys. Rep. 454, 1 (2007);

[32] M. Ablikim et al. (BESIII Collaboration), Phys. Rev. D 93, 112011 (2016).

[33] A. Etkin et al. Phys. Rev. Lett. 41, 784 (1978); Phys. Lett. B 165, 217 (1985); Phys. Lett. B 201, 568 (1988).

[34] Y. Chen et al., Phys. Rev. Lett. 111, 091601 (2013).

[35] M. Ablikim et al. (BESIII Collaboration), Phys. Rev. D 87, 092009 (2013). 